\title{
Effect of Open-Book Exercise Practice on Closed-Book Tests Results
}

\author{
Kyaw, $S$.
}

\begin{abstract}
Introduction: The present study was on 2nd MBBS (1/2011 batch) students at the University of Medicine-2, Yangon, Myanmar. Self-learning ability of students is gradually declining and students tend to neglect reading prescribed textbooks. Test marks have also declined considerably. Students find it difficult to identify salient points when answering questions. Thus, the present study aimed to study the effect of open-book exercise practice on closed-book tests results. Objectives were to identify the students' scoring of a closed-book written tutorial and to compare the changes in closed-book written tutorial marks after regular open-book study.
\end{abstract}

Methods: The study was carried out at the Department of Physiology, University of Medicine-2, Yangon, Myanmar for a period of one year. Students who attended the tutorial sessions were informed of the details of the study and 360 consented to participate. After serial interventions, results of 290 students involved in all tests were selected for analysis. In tutorial sessions, open-book questions and answers were studied by focusing on and critically analyzing the relevant chapter. The effectiveness was assessed by repeated closed-book tests after two or three chapters.

Results: The number of distinctions increased to $17 \%$ from baseline value (2\%). The number of students passing the test increased to $36 \%$ from baseline (12\%) and number of failing students decreased markedly to $47 \%$ from a baseline value of $86 \%$. The students' performance was significantly improved from baseline value after intervention $(p<0.001)$. Percentage change of marks was $15.40 \%$ (after $1 \mathrm{st}$ intervention), $17.47 \%$ (after 2nd intervention), 24.55\% (after 3rd intervention). Scoring percentage was highest in test-3.

Conclusion: Open-book exercise practices in tutorial sessions increase the marks in closed-book tests and improves the students' performance.

\section{Introduction}

At present, medical education has to deal with two important trends: a body of knowledge that is growing and changing faster than ever, and the increasing need to concentrate education on integration of knowledge, skills and attitudes. To fulfil these factors, open-book tests be a format that best suits these developments. The frequently used closedbook tests are suitable to assess the core knowledge, however, open-book tests seem more suitable to assess students' ability to manage backup knowledge.

Objectives of the present study were to determine student scores at closed-book written tutorials and to compare the changes in marks after practising regular open-book study.

Professor

Department of Physiology

University of Medicine 1

Yangon, Myanmar

E Mail: sandakyaw.myanmar@gmail.com

\section{Method}

This quasi-experimental study was conducted with the participation of second year MBBS students (1/2011 Batch) of the University of Medicine-2, Yangon, Myanmar, over a period of one year. Informed consent was obtained from all participating students. The intervention was undertaken during weekly tutorial sessions (3-6 hours per week), and all content was taught by system based study. At the end of each system, open book study questions were set up by the Head of Department and delivered to every tutorial session. Each tutorial session had about 30-35 students. Students were instructed to search relevant facts in the prescribed textbooks and note down the facts and answer the questions.

A total of 360 students consented to participate in this study. After serial interventions, the results of students who participated in all the tests were selected for analysis, with 290 students performing in all tests. During tutorial sessions, students responded to open-book 
questions and answers were learned by focused reading and critically reviewing the relevant chapters. The effectiveness was assessed by repeated closed-book tests after two or three chapters.

Closed-book test marks were collected to establish a baseline for student performance. The chapters involved were the autonomic nervous system, the excitable tissues, the cardiovascular system and the respiratory system. The baseline results indicated that our students need to improve in Physiology. After the intervention we did the first closed-book test on the gastrointestinal system and renal system; the second closed-book test on the endocrine system and reproductive system; and the third closed-book test on the skin and thermoregulatory system and the central nervous system.

\section{Results}

Criteria for distinction is 75 marks and above, with the pass mark at 50 of 290 students, 5 (2\%) obtained distinction passes, 35 (12\%) passed and 250 students (86\%) failed. After the 1st intervention, 34 students (12\%) obtained distinctions, 84 (29\%) passed and 172 (59\%) failed. After the 2nd intervention, 41 students (14\%) obtained distinctions, 84 (29\%) passed and 165 (57\%) failed. After the 3rd intervention, 48 students (17\%) obtained distinctions, 104 (36\%) passed and 138 (47\%) failed (Figure 1).

Figure 1: Results of tests performance

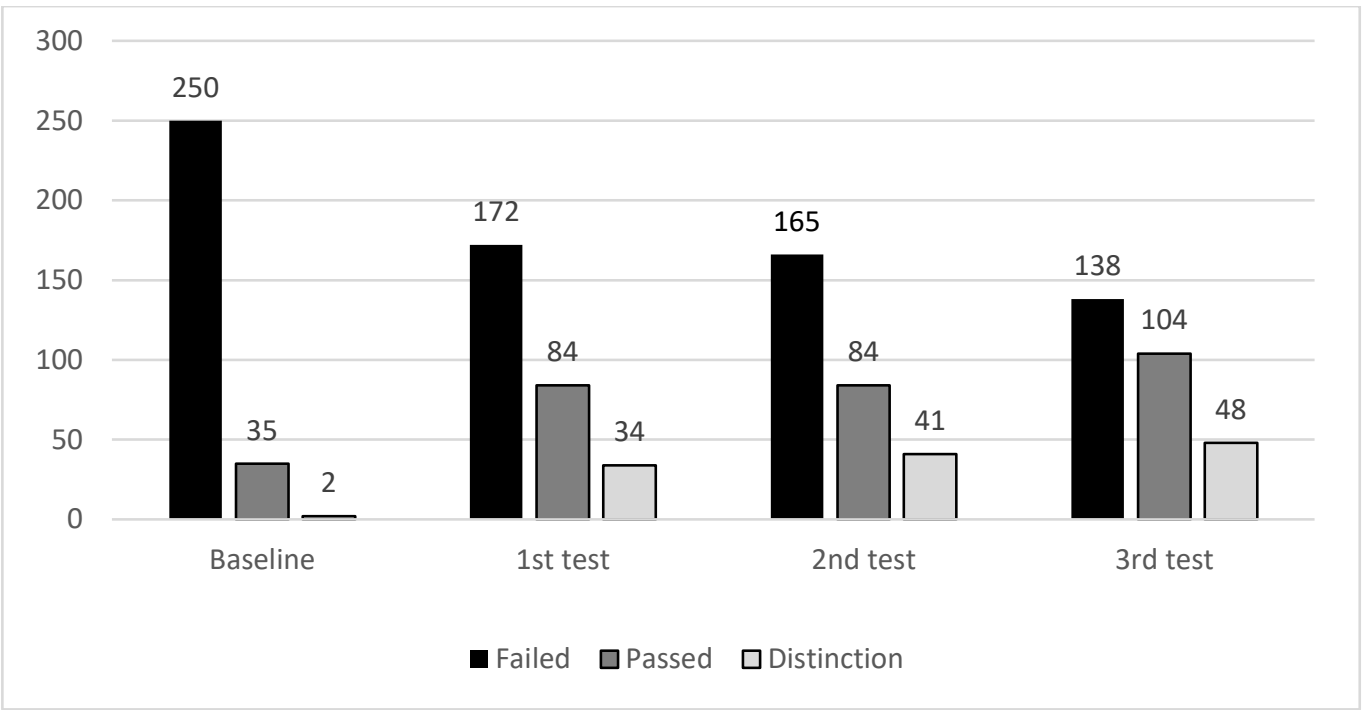

\section{Discussion}

Feller (1994) pointed out that closed book examinations only serve to demonstrate what students can do with whatever they have been able to memorize. The continued use of closed book examinations may encourage the students to live in the past rather than the future. Furthermore, the use of a closed book examinations only serves to test a student's ability to perform under very restrictive conditions. In this way, an open-book examination is able to almost completely replicate a real-world situation, (Loi \& Teo, 1999).

In 1999, Loi and Teo reported that students who prefer open-book examinations agree that such examinations will reduce the amount of time spent on preparation, make the learning process less stressful, require less memorization and allow more room for logical thinking. In the present study, although students' preference on open-book study tests was not identified, the performance was analyzed serially. The findings show a marked improvement before and after the intervention.

In 2008, Heijne-Penninga et al. also analyzed open and closed-book tests, and the psychometric quality of an assessment procedure with open and closed-book sessions was examined. The results showed that the use of open-book items alongside closed-book items is possible without much decrease in psychometric quality.

In the present study, improvement of performance was assessed by closed book tests. Although the present study did not assess 
the students' preference on tests, their test scores pointed out their predilection in learning.

\section{Conclusion}

Open-book exercises practiced in tutorial sessions increase the test scores in closedbook tests and improve the students' performance. Therefore, students need such training to achieve adult learning styles.

\section{Acknowledgements}

The present study was carried out in in 2011 as a component of the Diploma in Medical Education when the author served as a lecturer in the Department of Physiology, University of Medicine 2, Yangon. The author is grateful to Rector, Professor Tint Swe Latt, Dr. Daw Than
Oo, Head of Department of Physiology and colleagues from University of Medicine 2, for their kind help in conducting the study.

\section{References}

Feller, M. (1994) Open-book testing and education for the future, Studies in Educational Evaluation, 20, pp. 235-238.

Heijne-Penninga, M., Kuks, J.B.M., SchonrockAdema, J., Snijders, T.A.B. \& Cohen Schotanus, J. (2008) Open-book tests to complement assessment programmes: analysis of open and closed-book tests, Advances in Health Sciences' Education,13, pp. 263-273.

Loi, S.L. \& Teo, J.C.C. (1999) The Impact of Open Book Examinations on Student Learning, New Horizons in Education, 40, pp. 34-42. 